\title{
Results from chemoradiotherapy for squamous cell cervical cancer with or without intracavitary brachytherapy
}

\author{
Diogo Delgado, MD, André Figueiredo, MD, Vera Mendonça, MD, Marilia Jorge, MD, Miriam Abdulrehman, MD, \\ Maria Filomena de Pina, MD \\ Department of Radiation Oncology, Centro Hospitalar Universitário Lisboa Norte, Lisbon, Portugal
}

\begin{abstract}
Purpose: The aim of this study is to compare the outcomes of intracavitary high-dose-rate brachytherapy (BT-IC) boost and external beam radiotherapy (EBRT) boost in patients treated with concomitant chemoradiotherapy for squamous cell carcinoma of the cervix.

Material and methods: It is a retrospective review of 92 patients with stage IB1-IVA cervical cancer treated with concomitant chemoradiotherapy between 2008 and 2013. All patients received pelvic 3D conformal EBRT (range, 45-50.4 Gy) concomitant with weekly cisplatin $\left(40 \mathrm{mg} / \mathrm{m}^{2}\right)$, and a BT-IC boost (37 patients: 4 fractions of 6 Gy prescribed to a point $\mathrm{A}$ ) to the tumor or a 3D conformal EBRT boost (55 patients: $16.2 \mathrm{~Gy}$ ), if the former was not technically feasible.

Results: The 5-year overall survival and recurrence-free survival rates for both groups were $68 \%$ and $55 \%$, respectively. The 5-year overall survival and recurrence-free survival were better and statistically significant in the BT-IC group with $82 \%$ and $79 \%$, respectively, as compared to the EBRT group with $58 \%$ and $38 \%$, respectively. In multivariate analysis controlling for maximum tumor dimension, lymph node status, and FIGO stage, EBRT boost was associated with a statistical significant increase in the risk of recurrence (HR: 3.56; 95\% CI: 1.27-10.02; $p=0.016$ ) and a trend towards an increase in the risk of death (HR: 3.14; 95\% CI: 0.97-10.17; $p=0.056$ ). Lymph node status was also significantly associated with a greater risk of recurrence.

Conclusions: BT-IC boost was associated with a lower recurrence rate and better overall survival and recurrence-free survival. EBRT boost patients had a three-fold increase in the risk of recurrence. Brachytherapy is essential in the treatment of cervical cancer and improved alternatives are needed for patients who are not candidates for standard brachytherapy applicators.

Key words: high-dose-rate brachytherapy, cervical cancer, gynecological cancer, radiotherapy.

\section{Purpose}

Radiotherapy (RT) with concomitant chemotherapy is considered the standard treatment for locally advanced cervical cancer, and this treatment combination was compared to RT alone in randomized trials and showed an improved survival [1,2,3,4]. Guidelines recommend a combined RT treatment with external beam radiotherapy (EBRT) to the pelvis and intracavitary brachytherapy (low-, pulsed-, or high-dose-rate) boost to the tumor $[5,6]$. Intracavitary brachytherapy (IC-BT) allows for a highly conformal hypofractionated boost increasing the total tumor dose to over $80 \mathrm{~Gy}$ of $2 \mathrm{~Gy}$ equivalent dose $\left(\mathrm{EQD}_{2}\right)$, while maintaining acceptable doses to the nearby organs at risk. Large national databases and individual center retrospective reviews have suggested a benefit in outcomes when EBRT is combined with IC-BT $[7,8,9,10,11,12,13,14]$.
However, IC-BT is sometimes not technically possible, due to large tumor volume that cannot be fully covered without excessive toxicity to the organs at risk or difficulties concerning the technique, including an insertion of the intrauterine tandem. In such cases, EBRT tumor boost is given, usually up to 66 Gy. Because EBRT boost is not the ideal solution, the role of interstitial brachytherapy is recently growing in the treatment of locally advanced tumors.

The objective of this study was to compare the outcomes of patients treated with or without IC-BT boost at our department.

\section{Material and methods \\ Patient population}

This series includes 92 patients with histological diagnosis of squamous cell carcinoma of the cervix, treated 
Table 1. Patient and treatment characteristics of both treatment groups

\begin{tabular}{lccc} 
& $\begin{array}{c}\text { Brachytherapy } \\
(n=37)\end{array}$ & $\begin{array}{c}\text { EBRT } \\
(n=55)\end{array}$ & $P$ \\
\hline Patient & &
\end{tabular}

\begin{tabular}{lccc}
\hline Age, mean (years) & 53.7 & 53.4 & 0.903 \\
\hline MTD & 39.4 & 52.6 & $<0.0001$ \\
\hline FIGO stage (\%) & & & 0.004 \\
\hline
\end{tabular}

\begin{tabular}{lcc}
\hline IB1 & $1(2.7)$ & 0 \\
\hline IIA1 & $2(5.4)$ & 0 \\
\hline IIA2 & 0 & $3(5.5)$ \\
\hline IIB & $32(86.5)$ & $31(56.4)$ \\
\hline IIA & $1(2.7)$ & $4(7.3)$ \\
\hline IIIB & $1(2.7)$ & $14(25.5)$ \\
\hline IVA & 0 & $3(5.5)$ \\
\hline
\end{tabular}

\begin{tabular}{lccc}
\hline FIGO stage $(\%)$ & & & $<0.0001$ \\
\hline$\leq I I B$ & $35(94.6)$ & $34(61.8)$ & \\
\hline$>I I B$ & $2(5.4)$ & $21(38.2)$ & \\
\hline
\end{tabular}

\begin{tabular}{lccc}
\hline Lymph nodes (\%) & & & 0.161 \\
\hline Yes & $7(18.9)$ & $18(32.7)$ & \\
\hline No & $30(81.1)$ & $37(67.3)$ & \\
\hline
\end{tabular}

\begin{tabular}{lccc}
\hline Lymph node location (\%) & & & 0.355 \\
\hline Pelvic & $5(71.4)$ & $8(42.1)$ & \\
\hline Paraaortic & $1(14.3)$ & $1(5.3)$ & \\
\hline Both & $1(14.3)$ & $9(47.4)$ &
\end{tabular}

\begin{tabular}{lccc}
\hline Treatment & & & \\
\hline Pelvic EBRT dose (mean) & 49.7 & 50.3 & 0.051 \\
\hline Paraaortic EBRT (\%) & & & 0.306 \\
\hline Yes & $2(5.4)$ & $8(14.5)$ & \\
\hline No & $35(94.6)$ & $47(85.5)$ & \\
\hline
\end{tabular}

\begin{tabular}{lccl}
\hline Parametric EBRT boost (\%) & & & \\
\hline Yes & $26(70.3)$ & & \\
\hline No & $11(29.7)$ & & \\
\hline Boost EBRT dose (mean) & 10.5 & 16.2 & $<0.0001$
\end{tabular}

\begin{tabular}{|c|c|c|c|}
\hline \multicolumn{4}{|c|}{ Number of BT-IC fractions given (6 Gy) } \\
\hline 1 & $2(5.4)$ & & \\
\hline 2 & $3(8.1)$ & & \\
\hline 3 & $2(5.4)$ & & \\
\hline 4 & $30(81.1)$ & & \\
\hline BT-IC dose (mean) & 21.7 & & \\
\hline Total EQD 2 dose (mean) & 79.9 & 65.8 & $<0.0001$ \\
\hline Total BED dose (mean) & 95.9 & 78.9 & $<0.0001$ \\
\hline Treatment time (days) & 78 & 58.9 & $<0.0001$ \\
\hline
\end{tabular}

EBRT - external beam radiation therapy, BT-IC - intracavitary brachytherapy, FIGO - International Federation of Gynecology and Obstetrics, MTD - maximal tumor dimenson with concomitant chemoradiotherapy between 2008 and 2013 at a single radiotherapy department.

In 37 patients, pelvic EBRT was combined with a BTIC boost. The remaining 55 patients were treated only with EBRT because of technical feasibility. They received an EBRT boost to the tumor. The data was collected retrospectively from an investigation of patients records.

\section{Diagnosis and staging}

All patients underwent vaginal inspection, bimanual palpation, transvaginal ultrasound evaluation, and biopsies of the tumor. Most patients underwent radiological staging, usually a chest and abdomen contrast-enhanced computed tomography (CT) scan together with a gadolinium contrast-enhanced pelvic magnetic resonance imaging (MRI). Staging was completed in combination with clinical observation and radiological studies according to the International Federation of Gynecology and Obstetrics (FIGO) 2009 staging system.

\section{Brachytherapy}

BT-IC boost was given using a high-dose-rate iridium-192 ( ${ }^{192} \mathrm{Ir}$ ) source afterloader, with an intrauterine tandem and ring applicator set. Four fractions of 6 Gy were prescribed to point A, in most cases weekly, after the completion of pelvic EBRT. It was not possible to give the full four fractions to all patients receiving BTIC boost due to technical application constraints, usually difficulties in inserting the intrauterine tandem because of radiation stenosis of the cervical os or perforation (uterine or cervical). In those cases, an EBRT boost was given to complete the dose to the tumor. All patients who had at least one BT-IC fraction were included in the BT-IC group.

\section{External beam radiotherapy}

EBRT was given with 3D conformal radiotherapy (3D-CRT), using a linear accelerator with photon energies > $6 \mathrm{MV}$. CT simulation was made with a void rectum and filled bladder. All patients received pelvic field EBRT, with a clinical target volume (CTV) encompassing the tumor, cervix, uterus, upper third of the vagina, parametrial tissue, and regional lymph nodes (obturator, external iliac, internal iliac, presacral nodes, and common iliac nodes up to the level of $\mathrm{L}_{4}-\mathrm{L}_{5}$ ), with a median dose of 50.4 Gy (range, 45-50.4 Gy) in 1.8 Gy once-daily fractions. In case of clinically suspicious paraaortic lymph nodes detected on imaging, patients received paraaortic lymph node irradiation up to the level of $\mathrm{T}_{12}-\mathrm{L}_{1}$. If parametrial invasion was clinically palpable or detectable in imaging, patients who received the BT-IC boost were given a parametrial boost up to $16.2 \mathrm{~Gy}$ at the discretion of treating physician. The parametrial boost was only delivered after all brachytherapy applications to consider the dose already received to point B. In the EBRT only group, patients received a new treatment plan, with repeated CT simulation and clinical examination in the last week of treatment. The new CTV encompassed only the tumor, cervix, and uterus, with a median dose of 16.2 Gy 
(range, 7.2-21.6 Gy). No patients received EBRT boost to radiologically suspicious lymph nodes.

\section{Chemotherapy}

All patients received concomitant chemotherapy, with $40 \mathrm{mg} / \mathrm{m}^{2}$ of cisplatin given once a week.

\section{Follow-up}

Follow-up visits were made every 3 months for the first 6 months, then every 6 months for the first five years, and annually thereafter. Follow-up consisted of examination and annual cervical cytology. When a recurrence was suspected, a pelvic MRI or CT scan and a biopsy were performed for confirmation.

\section{Recurrence}

Local recurrence was defined as the absence of complete remission on gynecological examination, imaging, or a positive cervical cytology or biopsy. Regional recurrence included all relapses in pelvic and paraaortic lymph nodes detected by imaging or pathology. Distant recurrence was defined as distant organ spread.

\section{Statistics}

The Pearson chi-square test was used for differences in proportions and the Student $t$-test for comparison of mean variables of independent variables. The Kaplan-Meier method was used for survival analysis, comparing different curves with the log-rank test. Cox proportional hazard regression analysis was applied to run univariate and multivariate models to detect prognostic factors for survival. A $p<0.05$ was considered statistically significant. Follow-up was measured from date of diagnosis to date of event. Statistical analysis was made using IBM ${ }^{\circledR}$ SPSS $^{\circledR}$ Statistics Version 23.0 (IBM Corp., Armonk, NY, USA).

\section{Results}

\section{Patient groups characteristics}

The mean age of all patients was 53.5 years and it was similar across the two groups (Table 1). Patients from the EBRT boost group had larger tumors and more advanced disease at diagnosis; however, roughly the same number of patients had stage $\leq$ IIB ( 34 vs. 35 patients). There was no difference regarding the presence of clinically suspicious lymph nodes. In the BT-IC group, most lymph nodes were detected in the pelvic region $(71.4 \%)$, whereas in the EBRT group, most patients had both paraaortic and pelvic lymph nodes at diagnosis $(47.4 \%)$. Although there was no difference in pelvic EBRT dose between groups, there was a significant difference in total radiation dose and treatment time. In the BT-IC group, seven patients $(18.9 \%)$ did not complete the full four fractions prescribed, with two patients receiving one fraction only.

\section{Recurrence}

The overall recurrence rate for both groups was 34.8\% $(n=32)$. The EBRT boost group had a significantly higher
Table 2. Recurrence per treatment group and lymph node status

\begin{tabular}{lccc} 
& $\begin{array}{c}\text { Brachytherapy } \\
(n=37)\end{array}$ & $\begin{array}{c}\text { EBRT } \\
(n=55)\end{array}$ & $P$ \\
\hline Recurrence & $5(13.5)$ & $27(49.1)$ & 0.001 \\
\hline All & $4(12.5)$ & $12(37.5)$ & 0.653 \\
\hline Local & 0 & $5(15.6)$ & \\
\hline Regional (all paraaortic) & $1(3.1)$ & $10(31.3)$ & \\
\hline Distant & & \\
\hline Recurrence per lymph node status & & \\
\hline N0 $(n=67)$ & $14(20.9)$ & \\
\hline N1 $(n=25)$ & $18(72.0)$ & \\
\end{tabular}

recurrence rate compared to the BT-IC group (49.1\% vs. $13.5 \% ; p=0.001$ ) (Table 2). Most recurrences were local in both groups and more frequent in the EBRT group $37.5 \%$ vs. $12.5 \%$ ). Paraaortic lymph node recurrences were detected only on the EBRT group, in $15.6 \%$ of cases. Distant recurrences were rare in the BT-IC group compared to the EBRT group (3.1\% vs. $31.3 \%)$. Patients with clinically suspicious lymph nodes at diagnosis had a higher rate of recurrence compared to patients without regional disease (72\% vs. $20.9 \% ; p<0.0001$ ). Most patients with positive lymph nodes developed distant recurrences (56.3\%).

\section{Treatment time and total treatment dose}

Treatment time in the BT-IC group included all radiotherapy treatment, like pelvic EBRT, brachytherapy applications, and the boost to parametrial tissue if indicated, resulted in a longer overall treatment time compared with the EBRT only approach (78 days vs. 58.9 days; $p<0.0001$ ) (Table 1).

Total treatment dose converted to $\mathrm{EQD}_{2}$ and $\mathrm{BED}$ was significantly higher in the BT-IC group compared with the EBRT only group $\left(\mathrm{EQD}_{2}: 79.5 \mathrm{~Gy}\right.$ vs. $65.8 \mathrm{~Gy}$; $p<0.0001$ ) (BED: 95.9 Gy vs. 78.9 Gy; $p<0.0001$ ).

\section{Survival}

Median follow-up time for all patients was 67 months (range, 5-144 months).

The 5-year overall survival (OS) rate for both groups was $68 \%$. The BT-IC group had a statistically significant superior 5-year OS (82\% vs. 58\%; log-rank $p=0.005$ ) (Figure 1). Mean time for recurrence was 21.3 months. The 5 -year recurrence-free survival (RFS) rate was 55\% for both groups. The BT-IC group had also a superior 5-year RFS compared to the EBRT group (79\% vs. $38 \%$; log-rank $p<0.0001$ ) (Figure 2).

On multivariate Cox proportional regression analysis for RFS when controlling for maximal tumor dimension and FIGO stage, both clinically positive lymph node disease and treatment group (HR: 3.56; 95\% CI: 1.27-10.02; $p=0.016$ ) were statistically significant prognostic factors (Table 3). On multivariate analysis for OS, FIGO stage ( $\leq$ IIB vs. > IIB) was a significant prognostic factor, where- 


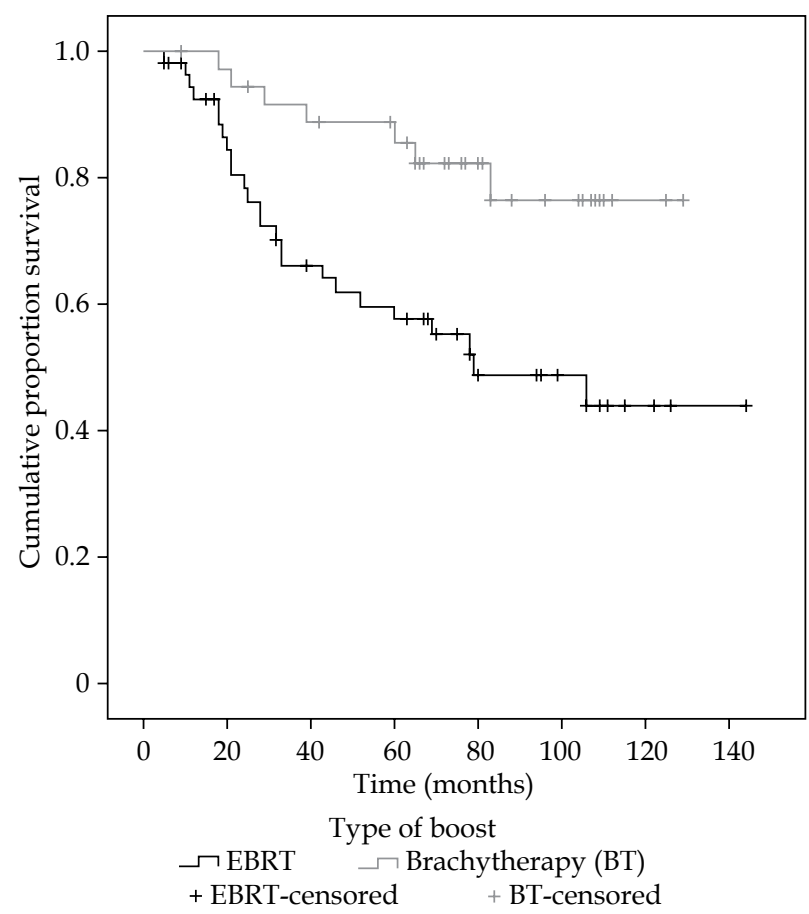

Fig. 1. Recurrence-free survival rate by the type of treatment boost delivered

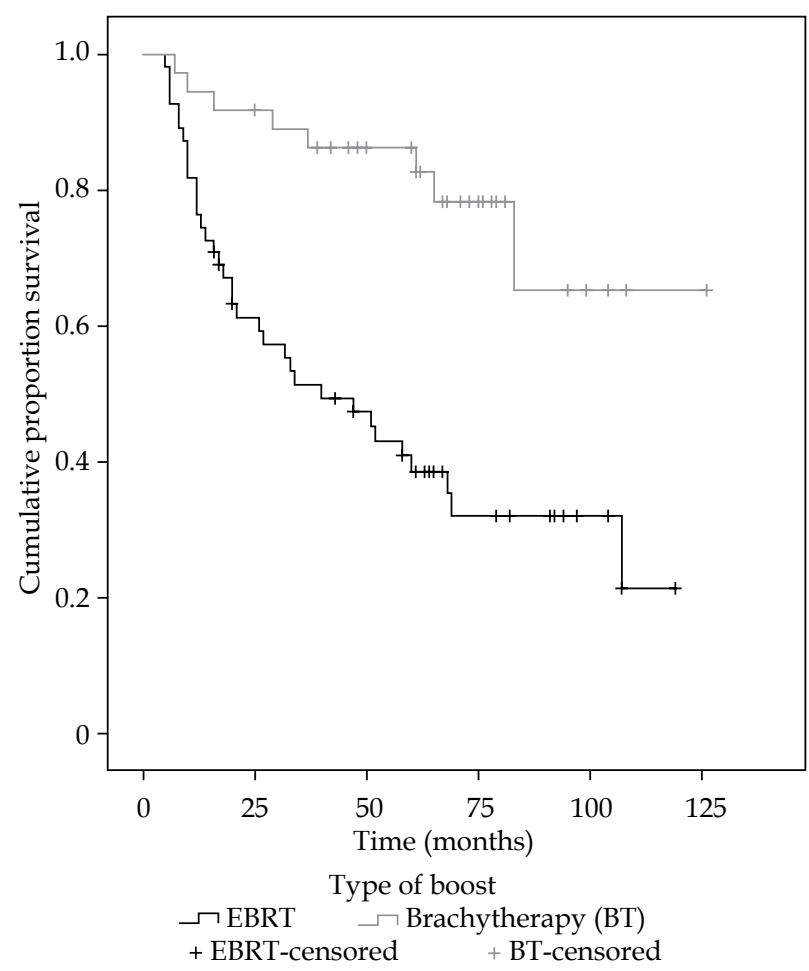

Fig. 2. Overall survival rate by the type of treatment boost delivered

as the treatment group displayed a clear trend towards an increase in the risk of death (HR: 3.14; 95\% CI: 0.97-10.17; $p=0.056$ ).

A further multivariate analysis included only patients with FIGO stage $\leq$ IIB from both treatment groups (EBRT 34 patients; BT-IC 35 patients). On multivariate Cox re- gression for RFS, the treatment group remained a statistical significant prognostic factor (Table 4), but not on multivariate regression for OS. Both lymph node status and maximal tumor dimension were not statistically significant in the analysis.

Table 3. Multivariate Cox proportional regression analysis for recurrence-free survival and overall survival

Multivariate Cox regression on RFS

\begin{tabular}{|c|c|c|c|c|}
\hline \multirow[b]{2}{*}{ Stage } & \multirow{2}{*}{$\frac{\mathrm{HR}}{0.693}$} & \multicolumn{2}{|c|}{$95 \% \mathrm{Cl}$} & \multirow{2}{*}{$\begin{array}{c}P \\
0.387\end{array}$} \\
\hline & & 0.302 & 1.59 & \\
\hline \multicolumn{5}{|c|}{$\leq \| \mathrm{IB}$ vs. $>\| \mathrm{B}$} \\
\hline Lymph nodes & 0.371 & 0.177 & 0.78 & 0.009 \\
\hline \multicolumn{5}{|c|}{ Negative vs. positive } \\
\hline MTD & 1.015 & 0.99 & 1.04 & 0.241 \\
\hline Boost & 3.564 & 1.268 & 10.02 & 0.016 \\
\hline \multicolumn{5}{|l|}{ EBRT vs. BT } \\
\hline \multicolumn{5}{|c|}{ Multivariate Cox regression on OS } \\
\hline & $\mathrm{HR}$ & \multicolumn{2}{|c|}{$95 \% \mathrm{Cl}$} & $P$ \\
\hline Stage & 0.347 & 0.136 & 0.885 & 0.027 \\
\hline \multicolumn{5}{|c|}{$\leq \|$ B vs. $>\| B$} \\
\hline Lymph nodes & 0.579 & 0.241 & 1.387 & 0.22 \\
\hline \multicolumn{5}{|c|}{ Negative vs. positive } \\
\hline MTD & 0.999 & 0.968 & 1.031 & 0.93 \\
\hline Boost & 3.14 & 0.969 & 10.171 & 0.056 \\
\hline \multicolumn{5}{|l|}{ EBRT vs. BT } \\
\hline
\end{tabular}

Table 4. Multivariate Cox proportional regression analysis for recurrence-free survival and overall survival on patients with FIGO stage $\leq \mathrm{IIB}$

Multivariate Cox regression on RFS

\begin{tabular}{|c|c|c|c|c|}
\hline & HR & \multicolumn{2}{|c|}{$95 \% \mathrm{Cl}$} & $P$ \\
\hline Lymph nodes & 0.466 & 0.181 & 1.2 & 0.114 \\
\hline \multicolumn{5}{|c|}{ Negative vs. positive } \\
\hline MTD & 1.023 & 0.994 & 1.052 & 0.121 \\
\hline Boost & 3.087 & 1.047 & 9.102 & 0.041 \\
\hline \multicolumn{5}{|l|}{ EBRT vs. BT } \\
\hline \multicolumn{5}{|c|}{ Multivariate Cox regression on OS } \\
\hline & $\mathrm{HR}$ & \multicolumn{2}{|c|}{$95 \% \mathrm{Cl}$} & $P$ \\
\hline Lymph nodes & 0.958 & 0.254 & 3.615 & 0.95 \\
\hline \multicolumn{5}{|c|}{ Negative vs. positive } \\
\hline MTD & 1.004 & 0.964 & 1.046 & 0.852 \\
\hline Boost & 2.826 & 0.817 & 9.775 & 0.101 \\
\hline EBRT vs. BT & & & & \\
\hline
\end{tabular}

MTD - maximal tumor dimenson, EBRT - external beam radiotherapy, $B T$ - brachytherapy 


\section{Discussion}

The 5-year OS rate $(68 \%)$ was higher than in other published series, especially the OS rate in the BT-IC group (82\%). As for the EBRT boost group OS results, although they were significantly lower than in the BT-IC group $(58 \%)$, the outcomes are still higher compared to other published results. Two Japanese groups reported their outcomes on patients treated with an EBRT boost, with a corresponding 3-year OS rate of $43.8 \%$ [15] and 2-year OS rate of $43 \%$ [16].

Possible contributions to these positive outcomes are the lower median age of the patient population (53 years), the use of concurrent chemotherapy in all patients, and the predominance of lower FIGO stage ( $\leq$ IIB).

Limitations of our study included its retrospective nature and differences in tumor size and FIGO stage between groups. As it was expected, most patients from the EBRT boost group had larger tumors and/or more advance disease at diagnosis, precluding them from BTIC. Also, some patients could receive only one to three fractions of BT-IC of the four prescribed, and completed the dose with EBRT. These patients were included in the BT-IC group, possibly resulting in bias regarding their outcome. In an attempt to minimize such biases, multivariate Cox regressions were performed, with maximal tumor dimension and FIGO stage as factors to decrease their contribution to RFS and OS outcomes. As the number of patients with lower FIGO stage was similar between groups, an additional analysis was performed only for patients with FIGO stage $\leq$ IIB. In both cases, the treatment group was a significant prognostic factor in terms of RFS, with a three-fold increase in the risk of recurrence. When all patients were considered, there was borderline statistical significance in the risk of death between EBRT and BT-IC.

Lanciano et al. reported results from patterns of care studies from the $70^{\prime}$ demonstrating a benefit for BT-IC in terms of pelvic control and survival [7]. Longson et al. published a retrospective review of 983 FIGO IIIB patients, also showing a benefit for BT-IC in terms of disease-free survival [8].

Karlsson et al. reviewed 220 patients, of which 134 had received a BT-IC boost, whereas 86 patients received EBRT boost alone. The 5-year OS and cancer-specific survival rates were $42.5 \%$ and $55.5 \%$, respectively, and all types of survival were better for patients receiving BT-IC [11].

Both groups had significant differences in total treatment time and total treatment dose. It is established that a shorter treatment time correlates with better outcomes $[17,18,19]$, and an overall treatment duration within 8 weeks (56 days) is currently recommended $[5,20]$. Shorter treatment time in the EBRT boost group ( 58.9 vs. 78 days) is expected, since the BT-IC boost was usually performed after completing pelvic EBRT. It is then reasonable to assume that the addition of BT-IC boost is more relevant than the overall treatment time, when considering boost approaches. Nevertheless, we have more recently changed our treatment protocol, starting an BT-IC boost application before the completion of pelvic EBRT to shorten the overall treatment time.
Almost all patients in our study had at least pretreatment pelvic $\mathrm{CT}$, allowing to detect any suspicious pelvic and/or paraaortic lymph nodes. Patients with radiologically suspicious lymph nodes had more frequent recurrences, mostly metastatic, and node positive disease was an important prognostic factor when it came to RFS. The presence of metastatic regional disease in cervical cancer correlates with a worse prognosis [21,22]. However, the 2009 FIGO classification was based purely on clinical examination and did not include lymph node assessment. The new 2018 FIGO classification [20] contains lymph node positive status (IIIC), which will stratify survival of cervical cancer patients more accurately.

Results from the Surveillance, Epidemiology, and End Results (SEER) database reveled a decline in the use of brachytherapy for cervical cancer between 1988 (83\%) and 2009 (58\%), while clearly demonstrating a benefit from BTIC in terms of OS and RFS [9]. This trend is thought to be accompanied by an increase in the use of more conformal EBRT techniques in a boost delivery, such as intensity-modulated radiation therapy (IMRT) or stereotactic body radiation therapy (SBRT). Feasibility studies show that it is possible to adequately cover the target volume with SBRT while maintaining a similar dose distribution for critical organs [23]. However, questions remain about the radiobiological equivalency of both treatments, since brachytherapy is capable of delivering not only over $80 \mathrm{~Gy} \mathrm{EQD}_{2}$ to the periphery of the tumor, but also doses over $120 \mathrm{~Gy} \mathrm{EQD}_{2}$ to the central cervix [14]. Clinical results from SBRT boost are scarce and often contradictory. Using the National Cancer Database, Gill et al. reported inferior OS outcomes from IMRT and SBRT compared with BT-IC [10], while O'Donnell et al. described similar outcomes between SBRT and BT-IC [12].

The prospective multicenter EMBRACE study [24] demonstrated that the addition of MRI-guided adaptive brachytherapy according to GEC-ESTRO recommendations $[25,26]$ resulted in improved local control, even in larger tumors, without additional toxicity. However, traditional brachytherapy applicators are sometimes still unhelpful in the treatment of cervical cancer patients, because of tumor size, lateralization, or technical inability to perform an intracavitary application. Interstitial brachytherapy techniques guided by ultrasound imaging, either alone [27] or combined with intracavitary applicators, could possibly allow for more patients to receive this highly needed boost to the tumor.

\section{Conclusions}

Brachytherapy boost provided better locoregional control and better survival outcomes compared with EBRT boost. Therefore, brachytherapy is an essential component in the treatment of cervical cancer patients and all efforts should be made to include this technique in the treatment plan.

\section{Funding}

This research did not receive any specific grant from funding agencies in the public, commercial, or not-forprofit sectors. 


\section{Disclosure}

The authors report no conflict of interest.

\section{References}

1. Morris M, Eifel PJ, Lu J et al. Pelvic radiation with concurrent chemotherapy com- pared with pelvic and para-aortic radiation for high- risk cervical cancer. N Engl J Med 1999; 340: 1137-1143.

2. Rose PG, Bundy BN, Watkins EB et al. Concurrent cisplatin-based radiotherapy and chemotherapy for locally advanced cervical cancer. N Engl J Med 1999; 340: 1144-1153.

3. Keys HM, Bundy BN, Stehman FB et al. Cisplatin, radiation, and adjuvant hysterectomy compared with radiation and adjuvant hysterectomy for bulky stage IB cervical carcinoma. N Engl J Med 1999; 340: 1154-1161.

4. Vale C, Tierney JF, Stewart LA et al. Reducing uncertainties about the effects of chemoradiotherapy for cervical cancer: a systematic review and meta-analysis of individual patient data from 18 randomized trials. J Clin Oncol 2008; 26: 5802-5812.

5. National Comprehensive Cancer Network. Cervical Cancer (Version 3.2019). http://www.nccn.org/professionals/physician_gls/pdf/cervical.pdf (accessed: December 19, 2018).

6. Marth C, Landoni F, Mahner S et al. Cervical cancer: ESMO Clinical Practice Guidelines for diagnosis, treatment and follow-up. Ann Oncol 2017; 28 (suppl 4): iv72-iv83.

7. Lanciano RM, Won M, Coia LR et al. Pretreatment and treatment factors associated with improved outcome in squamous cell carcinoma of the uterine cervix: A final report of the 1973 and 1978 patterns of care studies. Int J Radiat Oncol Biol Phys 1991; 20: 667-676.

8. Logsdon MD, Eifel PJ. Figo IIIB squamous cell carcinoma of the cervix: An analysis of prognostic factors emphasizing the balance between external beam and intracavitary radiation therapy. Int J Radiat Oncol Biol Phys 1999; 43: 763-775.

9. Han K, Milosevic M, Fyles A. Trends in the utilization of brachytherapy in cervical cancer in the United States. Int J Radiat Oncol Biol Phys 2013; 87: 111-119.

10. Gill BS, Lin JF, Krivak TC et al. National cancer data base analysis of radiation therapy consolidation modality for cervical cancer: The impact of new technological advancements. Int J Radiat Oncol Biol Phys 2014; 90: 1083-1090.

11. Karlsson J, Dreifaldt AC, Mordhorst LB et al. Differences in outcome for cervical cancer patients treated with or without brachytherapy. Brachytherapy 2017; 16: 133-140.

12. O'Donnell B, Shiao JC, Pezzi TA et al. Stereotactic body radiation therapy, intensity-modulated radiation therapy, and brachytherapy boost modalities in invasive cervical cancer: a study of the National Cancer Data Base. Int J Gynecol Cancer 2018; 28: 563-574.

13. Robin TP, Amini A, Schefter TE et al. Disparities in standard of care treatment and associated survival decrement in patients with locally advanced cervical cancer. Gynecol Oncol 2016; 143: 319-325.

14. Tanderup K, Eifel PJ, Yashar CM et al. Curative radiation therapy for locally advanced cervical cancer: brachytherapy is NOT optional. Int J Radiat Oncol Biol Phys 2014; 88: 537-539.

15. Ito K, Shimizuguchi T, Karasawa K. Clinical outcomes following conventional external beam radiotherapy boost in Japanese patients with cervical cancer who are ineligible for intracavitary brachytherapy. Jpn J Clin Oncol 2019; 49: 270275.

16. Matsuura K, Okabe T, Fujita K et al. Clinical results of external beam radiotherapy alone with a concomitant boost program or with conventional fractionation for cervical cancer patients who did not receive intracavitary brachytherapy. J Radiat Res 2012; 53: 900-905.

17. Perez CA, Grigsby PW, Castro-Vita $\mathrm{H}$ et al. Carcinoma of the uterine cervix. I. Impact of prolongation of overall treatment time and timing of brachytherapy on outcome of radiation therapy. Int J Radiat Oncol Biol Phys 1995; 32: 1275-1288.

18. Lanciano RM, Pajak TF, Martz K et al. The influence of treatment time on outcome for squamous cell cancer of the uterine cervix treated with radiation: A patterns-of-care study. Int J Radiat Oncol Biol Phys 1993; 25: 391-397.

19. Song S, Rudra S, Hasselle MD et al. The effect of treatment time in locally advanced cervical cancer in the era of concurrent chemoradiotherapy. Cancer 2013; 119: 325-331.

20. Bhatla N, Aoki D, Nand D et al. Cancer of the cervix uteri. Int J Gynaecol Obstet 2018; 143: 22-36.

21. Wang SC, Lin LC, Kuo YT et al. Radiographic number of positive pelvic lymph nodes as a prognostic factor in cervical cancer treated with definitive concurrent chemoradiotherapy or intensity-modulated radiotherapy. Front Oncol 2018; 8: 546.

22. Tseng JY, Yen MS, Twu NF et al. Prognostic nomogram for overall survival in stage IIB-IVA cervical cancer patients treated with concurrent chemoradiotherapy. Am J Obstet Gynecol 2010; 202: 174.e1-7.

23. Cengiz M, Dogan A, Ozyigit G et al. Comparison of intracavitary brachytherapy and stereotactic body radiotherapy dose distribution for cervical cancer. Brachytherapy 2012; 11: 125-129.

24. Pötter R, Georg P, Dimopoulos JCA et al. Clinical outcome of protocol based image (MRI) guided adaptive brachytherapy combined with 3D conformal radiotherapy with or without chemotherapy in patients with locally advanced cervical cancer. Radiother Oncol 2011; 100: 116-123.

25. Haie-Meder C, Pötter R, Van Limbergen E et al. Recommendations from Gynaecological (GYN) GEC-ESTRO Working Group (I): concepts and terms in 3D image based 3D treatment planning in cervix cancer brachytherapy with emphasis on MRI assessment of GTV and CTV. Radiother Oncol 2005; 74: 235-245.

26. Pötter R, Haie-Meder C, Van Limbergen E et al. Recommendations from gynaecological (GYN) GEC ESTRO working group (II): concepts and terms in 3D image-based treatment planning in cervix cancer brachytherapy-3D dose volume parameters and aspects of 3D image-based anatomy, radiation physics, radiobiology. Radiother Oncol 2006; 78: 67-77.

27. Sharma DN, Rath GK, Thulkar $S$ et al. Use of transrectal ultrasound for high dose rate interstitial brachytherapy for patients of carcinoma of uterine cervix. J Ginecol Oncol 2010; 21: 12-17. 